\title{
The Pelvic Organ Prolapse Incontinence Sexual Questionnaire, IUGA-revised (PISQ-IR)
}

\author{
Rebecca G. Rogers • M. E. Espuña Pons
}

Received: 17 September 2012 / Accepted: 20 September 2012/Published online: 30 April 2013

(C) The International Urogynecological Association 2012

Pelvic floor disorders greatly affect the lives of women who suffer from them. Whereas objective measures of disease severity are essential in the evaluation of these disorders, measures of symptom severity, quality of life, and function provide a broader and more comprehensive representation of the impact these disorders have on women's lives. Furthermore, they are critical for evaluating the impact of treatment on day-to-day life. The use of questionnaires to assess outcomes in urogynecology has been increasing. Originally, subjective outcomes were often assessed by simply asking the patient what and how bothersome their symptoms were and whether symptoms had resolved and function returned after treatment. Although central to clinical care, the weaknesses of measuring subjective outcomes in this manner for research are obvious, including lack of reproducibility, bias on the part of the provider in response interpretation, and lack of ability to compare outcomes across individuals and over time.

To address these problems, standardized, multidimensional questionnaires are often used. In scale development, the goal is to develop a questionnaire that captures concerns that are central to both patients and providers, that measures concerns in a meaningful way, that is reproducible within individuals and across populations, and that is developed so that valid inferences

\section{R. G. Rogers $(\bowtie)$}

Department of Obstetrics and Gynecology and Surgery, University of New Mexico Health Sciences Center,

Albuquerque, NM 87110, USA

e-mail: RRogers@salud.unm.edu

M. E. Espuña Pons

Clinical Institute of Gynaecology, Obstetrics and Neonatology,

Hospital Clinic, University of Barcelona,

Villarroel 170,

Barcelona 08036, Spain with meaning to clinical and personal evaluation about status and function can be drawn [1]. In the past two decades, an array of valid and reliable condition-specific measures of pelvic floor function has been developed. These measures represented a large improvement over ad hoc questioning and are widely used in both research and clinical settings. Nonetheless, there is still much work to do to improve these tools, particularly with respect to their international use.

The Pelvic Organ Prolapse Incontinence Sexual Questionnaire (PISQ) is the primary measure used to assess sexual function in women with urinary incontinence and prolapse, yet the original measure had deficits $[2,3]$. The International Urinary Gynecological Association (IUGA) Sexual Function Working Group aimed to conduct a fullscale re-evaluation of the PISQ, with the goals of refining its measurement properties, enhancing the ability to assess outcomes in women who are not sexually active and in women with anal incontinence, and developing an instrument ultimately intended for international use [4]. These goals have been realized in the development of the PISQ-IR. The new measure is not just a refinement of items found in the PISQ but includes new approaches to assessing the inherent diversity of women with pelvic floor disorders (PFD), such as gender-neutral items, to assess the impact of partner on sexual function. Importantly, this study was designed with input from a team of urogynecologists from around the world, from item development to evaluation and determination of the content of the final scales, with the explicit goal of having an instrument that was directed toward international usage from inception.

In this issue of the International Urogynecology Journal, we present the validity, reliability, and responsiveness of this new measure: the PISQ-IR [5]. In a companion article, we further present data explaining PISQ-IR scale creation and 
scoring [6]. The measure is now ready for international validation, and a number of IUGA members have already begun validation studies in a variety of languages under the guidance of the IUGA Research Committee. Ultimately, our hope is that this international collaboration will lead to improved assessment of sexual function in women with PFDs, no matter where they live or what language they speak.

\section{References}

1. Rockwood T, Constantine M (2009) Item and instrument development to assess sexual function and satisfaction in outcomes research. Int Urogynecol J 20(suppl 1):S57-S64
2. Rogers RG, Kammerer-Doak D, Villarreal A, Coates K, Qualls C (2001) A new instrument to measure sexual function in women with urinary incontinence or pelvic organ prolapse. Am J Obstet Gynecol 184(4):552-558

3. Omotosho TB, Rogers RG (2009) Int Urogynecol J 20(1):S52-S56

4. Espuña Pons M (2009) Sexual health in women with pelvic floor disorders: measuring the sexual activity and function with questionnaires - a summary. Int Urogynecol J 20(suppl 1):S65S71

5. Rogers RG, Rockwood TH, Constantine M et al (2012) A new measure of sexual function in women with pelvic floor disorders (PFD); Pelvic Organ Prolapse/Incontinence Sexual Questionnaire, IUGA-Revised (PISQ-IR). Int Urogynecol J doi:10.1007/s00192012-2020-8

6. Rockwood TH, Constantine ML, Adegoke O et al (2012) The PISQIR: Considerations in scale scoring and development. Int Urogynecol J doi:10.1007/s00192-012-2037-z 